\title{
Novel free-form optical surface design with spiral symmetry
}

\author{
Pablo Zamora ${ }^{\text {a }}$, Pablo Benítez ${ }^{\text {a,b }}$, Juan C. Miñano ${ }^{\text {a,b }}$, Juan Vilaplana ${ }^{\text {b }}$ \\ ${ }^{\mathrm{a}}$ CEDINT, Technical University of Madrid (UPM), Campus de Montegancedo 28223 Pozuelo de \\ Alarcón, Madrid, Spain \\ ${ }^{\mathrm{b}}$ LPI-LLC, 2400 Lincoln Ave., Altadena, CA 91001, USA
}

\begin{abstract}
Manufacturing technologies as injection molding or embossing specify their production limits for minimum radii of the vertices or draft angle for demolding, for instance. These restrictions may limit the system optical efficiency or affect the generation of undesired artifacts on the illumination pattern when dealing with optical design. A novel manufacturing concept is presented here, in which the optical surfaces are not obtained from the usual revolution symmetry with respect to a central axis ( $z$ axis), but they are calculated as free-form surfaces describing a spiral trajectory around $z$ axis. The main advantage of this new concept lies in the manufacturing process: a molded piece can be easily separated from its mold just by applying a combination of rotational movement around axis $z$ and linear movement along axis $z$, even for negative draft angles. The general designing procedure will be described in detail.
\end{abstract}

Keywords: geometric optical design, manufacturing techniques, illumination, CPV

\section{INTRODUCTION}

When dealing with optical designs based on plastic refractive elements, the first idea and the simpler one is to develop continuous lenses. Nevertheless this type of solutions always implies two vital drawbacks, both of them related to the lens thickness. The first of them is an increase on the system global cost: the thicker the lens is the more material quantity we will need and the more expensive the system will be.
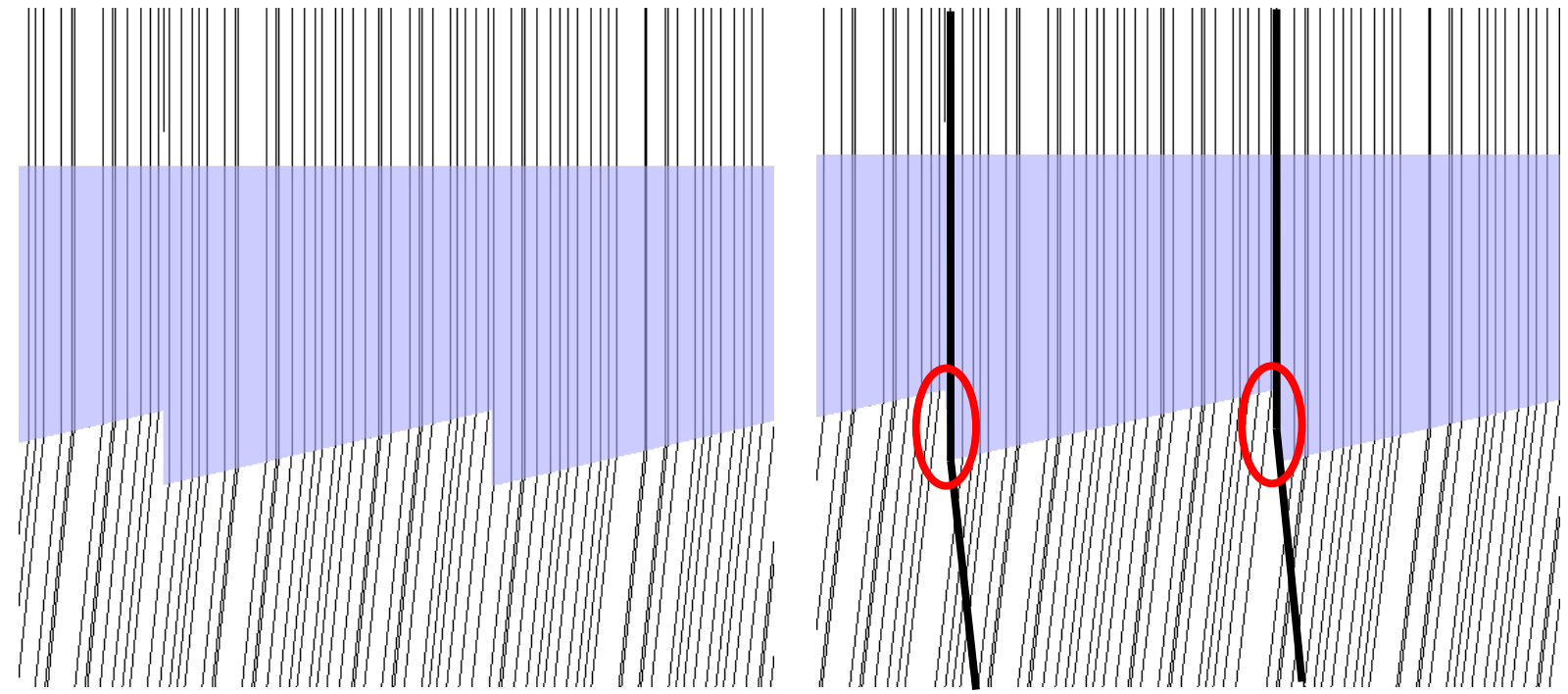

Figure 1 Optical efficiency losses due to draft angle. (Left) $0^{\circ}$ draft angle flat Fresnel lens, where every single normal incidence ray focuses on a single point at the focal distance. (Right) $4^{\circ}$ draft angle flat Fresnel lens, where bold black lines denote rays hitting draft angle and thus being wrongly directed. Red circle points at the draft angles where rays hit.

The second drawback has to deal with the manufacturing process itself [1]. If manufacturing a thick lens (over 10mm thick) by the well-known plastic injection process, it is really difficult a proper result, due to the nonuniform shrinkage of 
the material when it is cooling down. For instance, the PMMA dimensions shrink about $0.4 \%$ when solidifying, but the volume close to the surface of the piece cools down faster than de interior. If the piece has uniform thickness (faceted lenses, for example) the shrinkage is rather uniform. However, when thickness is non-uniform (continuous lenses case), the thinner parts solidify completely while the interior of thicker parts still have to shrink, resulting in sinks and deformations on the piece surface.

These two important problems lead us to choose solutions based on faceted optical elements such as Fresnel or TIR lenses. Nevertheless faceted elements have manufacturing constrains that limit their geometry, mainly due to the draft angle issue (see Figure 1). If single-piece manufacturing is desired, draft angles must have a positive value, higher than a limit (typically $>2^{\circ}$ ), in order to allow an unmolding process without frictions (these frictions can generate important lens deformations). If dealing with domed-shape elements negative draft angles are required so we can have an acceptable optical efficiency. In this case lenses must be built from several parts and join them all in a piece after having molded each one of the single parts, implying higher manufacturing complexity and cost.

The novel solution proposed here allows for a single-piece manufacturing process with zero or negative draft angles when designing faceted optical elements (mainly Fresnel lenses). These new designs are developed based on spiral symmetry (each facet converting into the adjacent one after each turn), instead of the classical revolution symmetry development (each facet describes a perfect ring after a whole turn around axis z). This freedom in draft angles value presented by spiral symmetry designs maximizes optical efficiency and avoids the light incidence on the corner radii by design.

\section{A BASIC SPIRAL EXAMPLE: FLAT FRESNEL LENS}

Let us begin explaining our method with the most basic example of faceted refractive optical element: the flat Fresnel lens. From now on we will work with the nomenclature and conventions introduced by concentration photovoltaics (CPV) instead of illumination to make explanations easier, even though this design can be used for both applications.

\subsection{Problem analysis}

The classical Fresnel lens couples a plane input wavefront (with -z direction) with a spherical output wavefront with origin in the focal plane center. For the flat Fresnel lens with spiral symmetry the input wavefront remains the same, but the output one changes slightly: the output rays must reach now a circular ring centered in the focal plane. This output wavefront will be helicoidal, presenting a vortex at its center (i.e. $z$ axis, where there will be no light).

Using conservation of étendue theorem in two dimensions [2], a wavefront must verify:

$$
d E_{2 D}=d x d p+d y d q+d z d r=0
$$

where $p, q$ and $r$ are the coordinates of the phase space corresponding to axis $x, y$ and $z$, respectively. In the simple case in which the receiver is a straight line (placed in the $x$ axis, thus $y=z=0$ ), according to conservation of étendue (2) must be satisfied:

$$
d E_{2 D}=d x d p=0
$$

In this situation, rays reaching the receiver will form an angle ( $\beta$ from now on) with respect to that straight line, being $\beta$ constant for a specific point of the receiver [3]. This means that every single ray impinging on the same point of the receiver, it will be placed over the surface of a cone with circular base whose axis of symmetry will be the receiving line. In our specific case in which the receiver is a circular ring, the line of symmetry of our cone will be the tangent line to the ring at each one of its points. Therefore, and similarly to the straight line case, for each specific point of the receiver every ray impinging on it will be placed on the surface of a cone forming an angle $\beta$ with the tangent line of the receiver. It is important to remark that angle $\beta$ will vary with the position of the receiver chosen. 


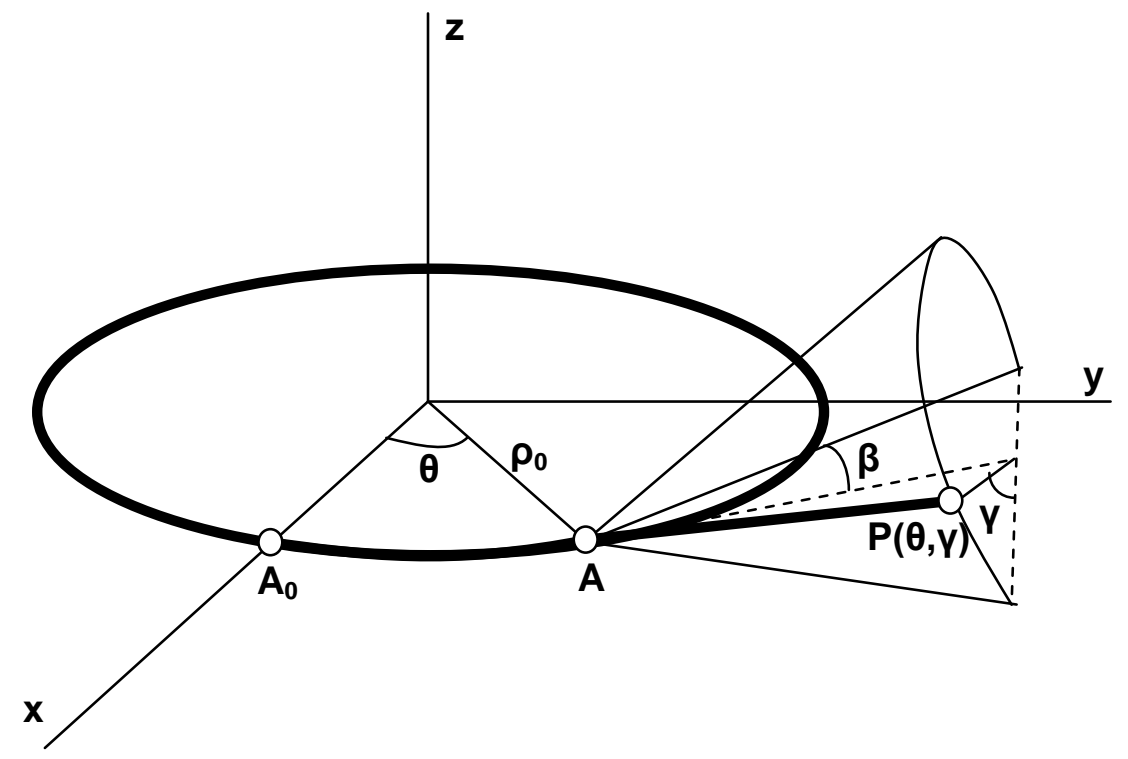

Figure 2 General scheme for the receiver ring, placed on $\mathrm{z}=0$ plane.

Figure 2 shows a canonical situation for our spiral Fresnel lens design. Point A belonging to circular-shaped receiver with constant radius $\rho_{0}$ has been chosen to illustrate what has been explained in the previous paragraph. As shown in the figure, $\theta$ is the angle of point A position respect to initial point $\mathrm{A}_{0}$ (placed on $x$ axis). Calculating the corresponding $\beta$ value for point $\mathrm{A}$, let us determine any point $\mathrm{P}$ in the $3 \mathrm{D}$ space just by knowing two angular parameters and a scalar one $\mathrm{P}(\theta, \gamma, d)$, instead of the classical Cartesian coordinate system $\mathrm{P}(\mathrm{x}, \mathrm{y}, \mathrm{z})$. $\theta$ determines the point on the receiver; $\gamma$ determines the corresponding line over the receiving cone; $d$ indicates the physical distance between $\mathrm{A}$ and $\mathrm{P}$ (and so the optical path length of raying going from $\mathrm{P}$ to $\mathrm{A}$ ).

If we consider the skew invariant concept [4], defined for this situation by:

$$
h=n \rho_{0} \cos \beta
$$

where $n$ is the refractive index, the analytical development of our problem provides a double solution. In the first one (with positive skew invariant $h>0$ ) the cone aperture is directed clockwise all along the receiver. In the second one $(h<0)$, the cone aperture is directed anti-clockwise. As a consequence, depending on $h$ sign, spiral direction of the designed lens will be clockwise or anti-clockwise.

\subsection{Spiral geometrical description}

We have already explained the general design characteristics for the spiral flat Fresnel lens. However, we can find a vast universe of different spiral geometry types (Archimedean, Fermat, logarithmic...) that our design can be based on. Let us identify which spiral of all possible ones match the stated conditions, i.e. ring-shaped receiver fulfilling a receiving cone with $\beta$ constant for every single point. Figure 3 shows a 3D analysis of the spiral line upon which the whole lens will be generated. The receiver is placed at $z=0$ plane, while the generating spiral is placed in a higher physical place. An analysis for generic point $\mathrm{P}$ will be done.

Unitary vector $\hat{t}$ is tangent to spiral line in point P. From geometrical deduction in Figure $3 \hat{t}=-\hat{Z} \times \hat{r}$, implying that $\hat{t}$, and thus the whole spiral line, will be contained in a $z=z_{0}=$ constant plane. In this situation we can build the surface of the lower part of the lens starting by this spiral line, which will be placed at the lowest part of the lens. The upper lens surface will be flat, so no calculation is needed. 


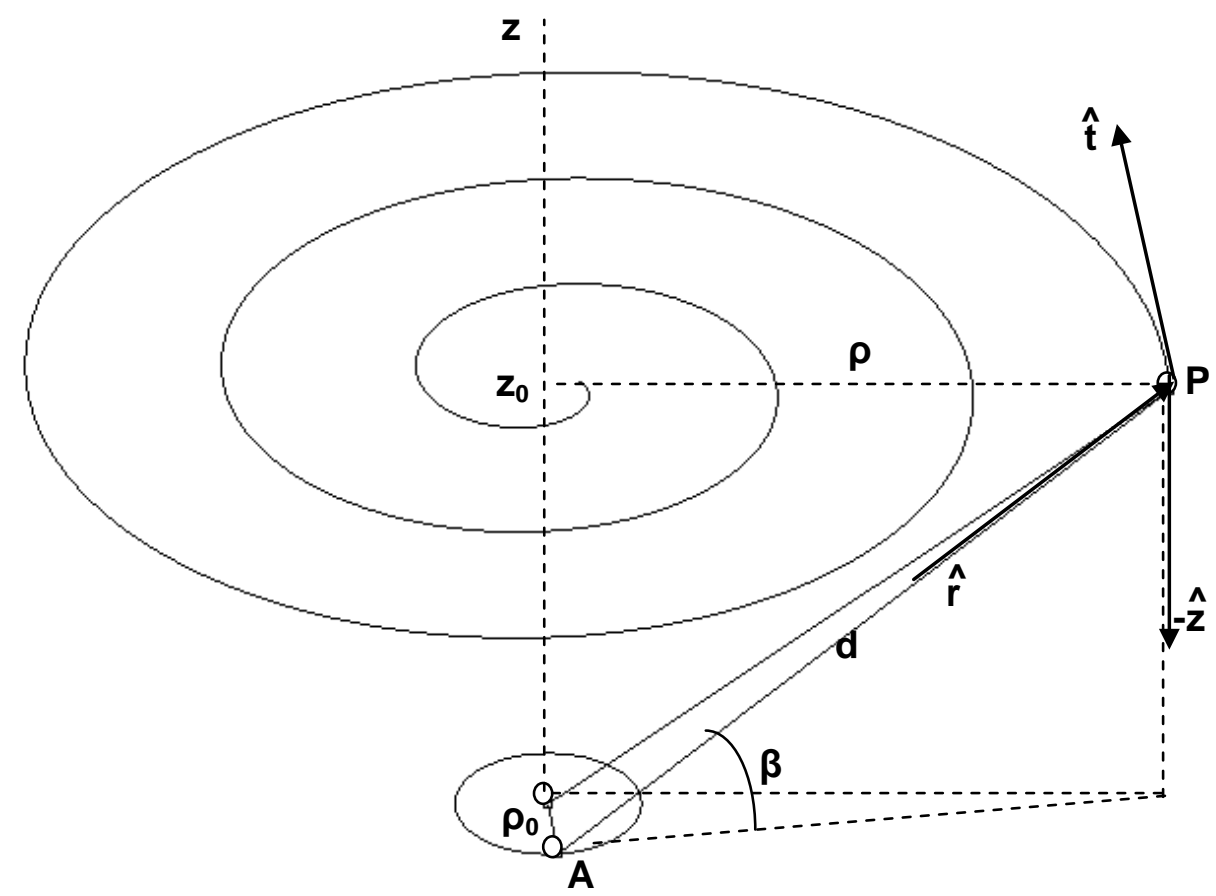

Figure 3 Scheme with generating spiral at $\mathrm{z}=\mathrm{z}_{0}$ plane and receiver at $\mathrm{z}=0$ plane for a generic spiral point $\mathrm{P}$

The shape of a particular 2D spiral is perfectly determined by defining the dependence, for any point belonging to it, between its angular parameter $\theta$ and its distance to the spiral geometrical center, $\rho$. Figure 4 offers a 2D simplified version of Figure 3 for an easier understanding of the analysis and easier identifying of the these parameters. We can deduce from Figure 4:

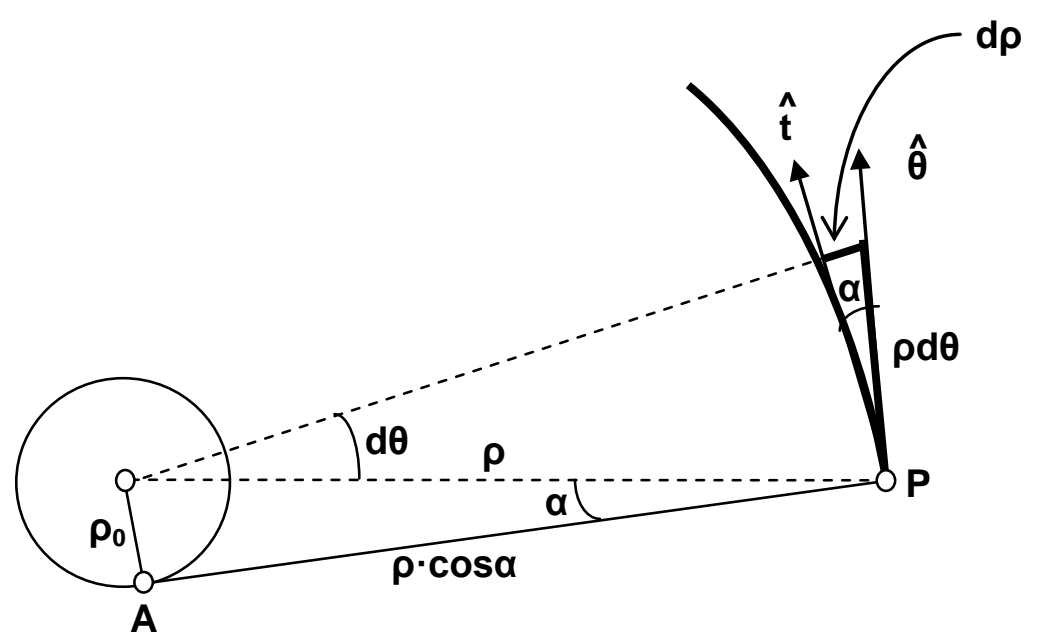

Figure 4 View from above (projection on $\mathrm{z}=$ constant plane) of Figure 2 


$$
\left.\begin{array}{l}
\sin [\alpha(\rho)]=\frac{\rho_{0}}{\rho} \\
\tan [\alpha(\rho)]=\frac{d \rho}{\rho d \theta}
\end{array}\right\} \tan \left[\arcsin \left(\frac{\rho_{0}}{\rho}\right)\right]=\frac{d \rho}{\rho d \theta} \Rightarrow \int d \theta=\int \frac{d \rho}{\rho \cdot \tan \left[\arcsin \left(\frac{\rho_{0}}{\rho}\right)\right]}
$$

Taking into account that almost the whole surface of lens will be composed by points for which approximation $\rho_{0}<<\rho$ is very reasonable, we can obtain:

$$
\int d \theta \simeq \int \frac{d \rho}{\rho_{0}} \Rightarrow \rho(\theta)=K+\rho_{0} \cdot \theta
$$

where $K$ is a constant. Equation (5) describes a well-known curve called involute of a circle, whose graphical representation is shown in Figure 5. It is important to point out that $\theta$, expressed in radians, can take values over to $2 \pi$. Each turn around the generating circle, which means an angular variation of $\theta=2 \pi$, produces a $2 \pi \rho_{0}$ increase in $\rho$ value, being $\rho_{0}$ the circle radius. $K$ value allows starting to build the involute in the desired point of the generating circle. This involute describes the solution to our problem if we project the generating spiral on the $z=0$ plane (same plane than receiver), being this situation identical to that shown in Figure 5.

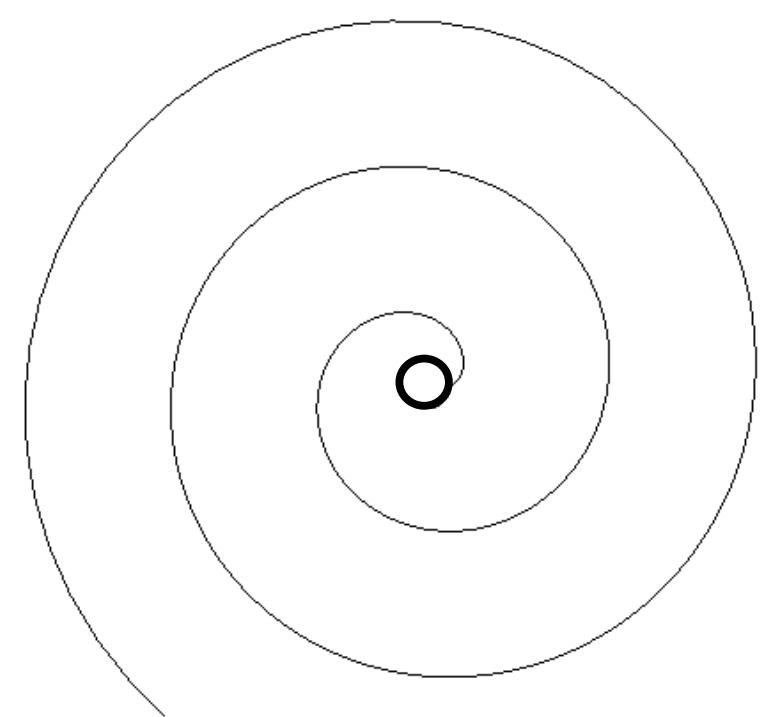

Figure 5 Involute of a circle (generating circle in bold line)

Analyzing Figure 3, again with $\rho_{0}<<\rho$ approximation, and applying the result calculated in equation (5), we can obtain the next equation, which describes $\beta$ value for every single point of the receiver, depending on its position $(\theta)$ :

$$
\tan \beta=\frac{z_{0}}{\rho_{0} \cdot \theta}
$$

Resulting equation (6) will allow us to build the lens surface easily, as shown in next chapter. 


\subsection{Lens constructions}

We will build the whole lens starting by the generating spiral. In order to make the calculation easier, we will express all the equations in terms of three parameters, as suggested in chapter 2.1: $\theta, \gamma, d$ (see Figure 2). In this way, we can reach every single point in the whole $3 \mathrm{D}$ space, $P(\theta, \gamma, d)$ by applying:

$$
\bar{P}(\theta, \gamma, d)=\bar{A}(\theta)+d \cdot \hat{r}(\theta, \gamma)
$$

where every variable has already explained in chapter 2.1. Let us express all the variables in function of the desired parameters:

$$
\begin{array}{r}
\bar{A}(\theta)=\left(\rho_{0} \cos \theta, \rho_{0} \sin \theta, 0\right) \\
\hat{r}(\theta=0)=\hat{r_{0}}=\left(\begin{array}{l}
r_{0 x} \\
r_{0 y} \\
r_{0 z}
\end{array}\right)=\left(\begin{array}{c}
\sin \beta \cos \gamma \\
\cos \beta \\
\sin \beta \sin \gamma
\end{array}\right) \\
\hat{r}(\theta, \gamma)=\left(\begin{array}{c}
r_{x} \\
r_{y} \\
r_{z}
\end{array}\right)=\left(\begin{array}{ccc}
\cos \theta & -\sin \theta & 0 \\
\sin \theta & \cos \theta & 0 \\
0 & 0 & 1
\end{array}\right) \cdot\left(\begin{array}{l}
r_{0 x} \\
r_{0 y} \\
r_{0 z}
\end{array}\right)=\left(\begin{array}{c}
\sin \beta \cos \gamma \cos \theta-\cos \beta \sin \theta \\
\sin \beta \cos \gamma \sin \theta+\cos \beta \cos \theta \\
\sin \beta \sin \gamma
\end{array}\right)
\end{array}
$$

leading to the next expression:

$$
\bar{P}(\theta, \gamma, d)=\left(\begin{array}{c}
x(\theta, \gamma, d) \\
y(\theta, \gamma, d) \\
z(\theta, \gamma, d)
\end{array}\right)=\left(\begin{array}{c}
\rho_{0} \cos \theta+d(\sin \beta \cos \gamma \cos \theta-\cos \beta \sin \theta) \\
\rho_{0} \sin \theta+d(\sin \beta \cos \gamma \sin \theta+\cos \beta \cos \theta) \\
d \sin \beta \sin \gamma
\end{array}\right)
$$

Now we will show that, to render our analysis even simpler, we can reduce point $\mathrm{P}$ dependence to just two parameters, $\theta$ and $\gamma$. Even though the lens presents a spiral symmetry, since it couples an input wavefront with an output one, the lens lower surface will be a Cartesian oval and thus an extra condition can be imposed. This condition is that of all normal incident rays impinging on the lens aperture area and arriving to receiver must have constant value of optical path length. In this way, using the eikonal function [5], $L$, we can obtain the optical path length between points A and P just by subtracting both points' eikonal function values. Using this extra condition and equation (6) we arrive to the following expression of distance $d$, applicable to all the points belonging to lens lower surface (we just need to substitute $\beta$ by its value calculated with (6)):

$$
d(\theta, \gamma, \beta)=\frac{\sqrt{\left(\rho_{0} \cdot \theta\right)^{2}+z_{0}{ }^{2}} \cdot(1-n \sin \beta)}{1-n \sin \beta \sin \gamma}
$$

being $n$ the material refraction index, and $\sqrt{\left(\rho_{0} \cdot \theta\right)^{2}+z_{0}^{2}}$ the value of $d(\theta, \gamma)$ when $\gamma=\pi / 2$ (i.e. projection of line between $A$ and $P$ on $z=0$ plane is tangent to generating circle in point A). If (6) is applied to (10) and (10) to (9) we can finally obtain the desired expression $P(\theta, \gamma)$ for the points belonging to the lens lower surface. 
Now it can be deduced that the generating spiral line, placed on the plane $z=z_{0}$ (Figure 3), is obtained for all those points satisfying $P(\theta, \pi / 2)$. Starting by values $\gamma=\pi / 2$ and $\theta=0$ (i.e. $\beta=\pi / 2$ ) we can vary progressively vary $\theta$ to higher values in order to describe the whole generating spiral line. If we additionally vary $\gamma$ as well, lens surface will also be built. In this process, we will design the lens along "several turns" (ranging $\theta$ in more than one turn) and then we will cut our lens by a vertical wall generated by a vertical extrusion of our spiral curve (creating the inactive facets). Figure 6 shows only active facets, while Figure 7 shows our lens already cut, with the vertical facets dividing

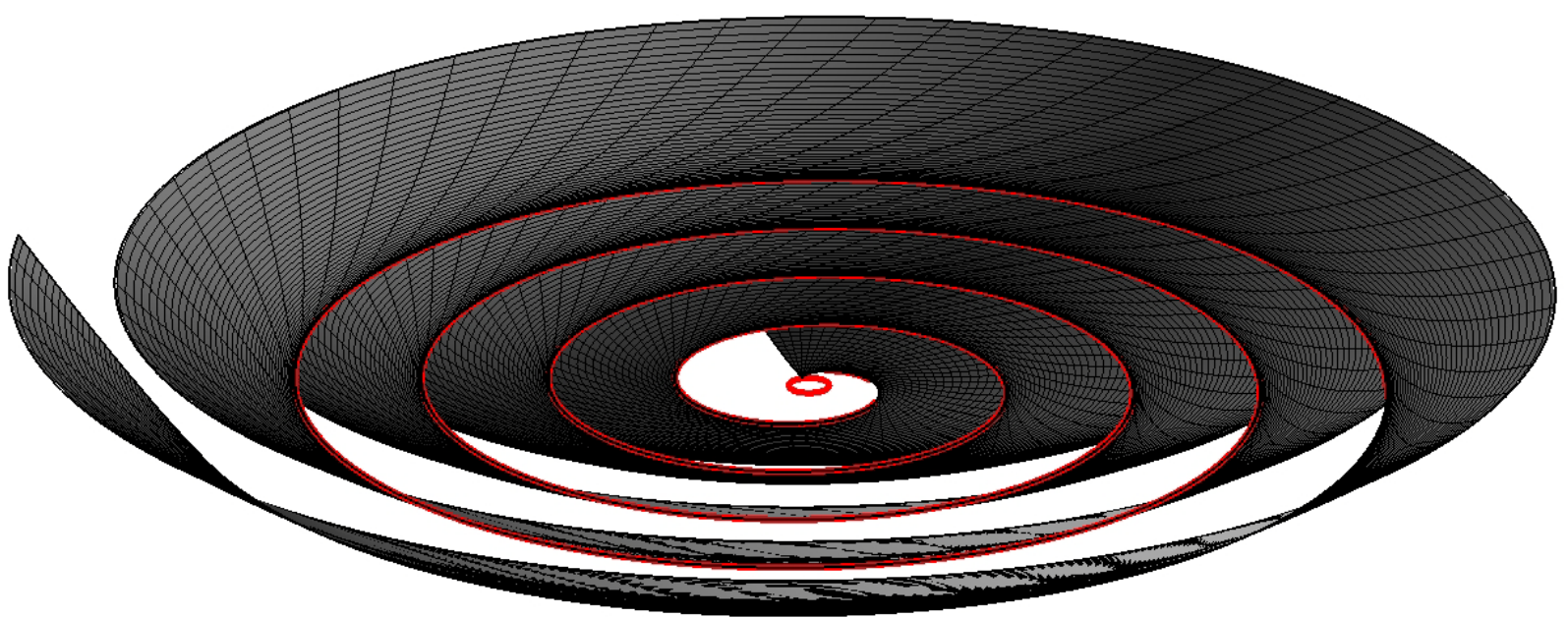

Figure 6 Several turns of the lens active surface (generating spiral in red color).

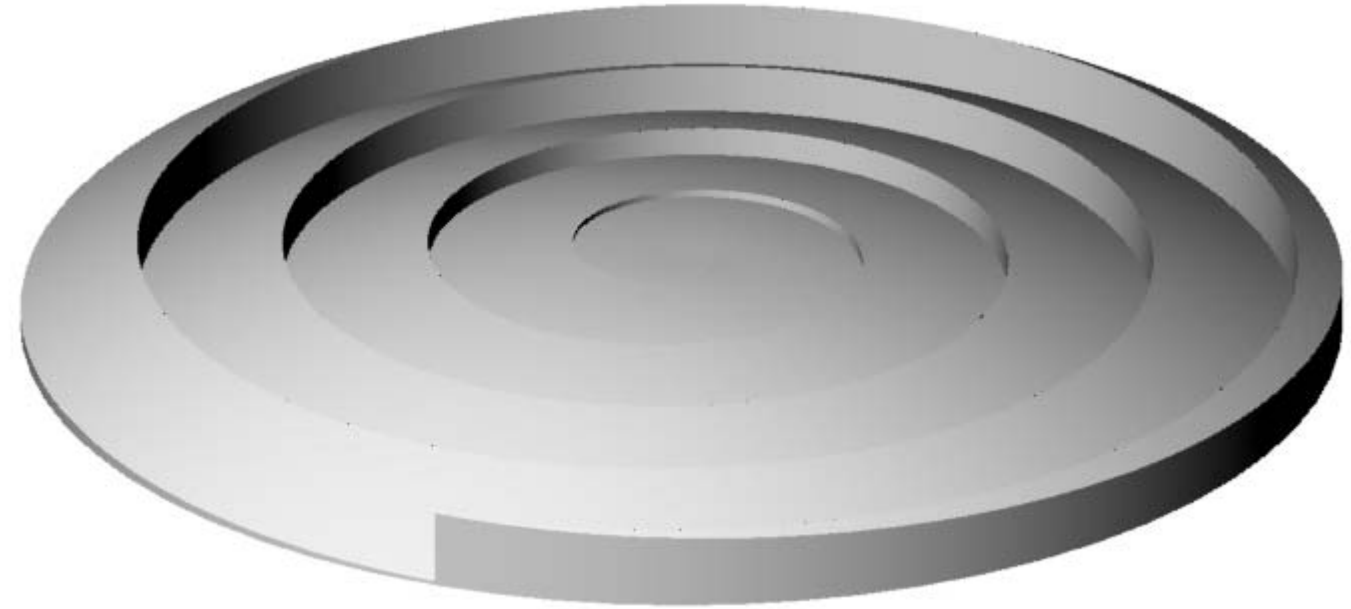

Figure 7 Lens already cut with vertical facets.

\subsection{Simulation results}

Raytrace simulations have been carried out in order to compare a flat Fresnel lens developed with spiral symmetry and a classical flat Fresnel lens presenting revolution symmetry. We have chosen refractive index of PMMA and a f/1.5 parameter for both, while $2^{\circ}$ draft angles have been taken into account for the revolution symmetry lens, which is a quite realistic value. Efficiency results show a significant relative gain of $1.6 \%$ when working with the spiral design $(91.9 \%$ 
optical efficiency for spiral design vs. $90.4 \%$ for revolution symmetry one, monochromatic $\lambda=555 \mathrm{~nm}$ simulations). Figure 8 shows both illumination patterns, one spot-shaped and the other one ring-shaped.
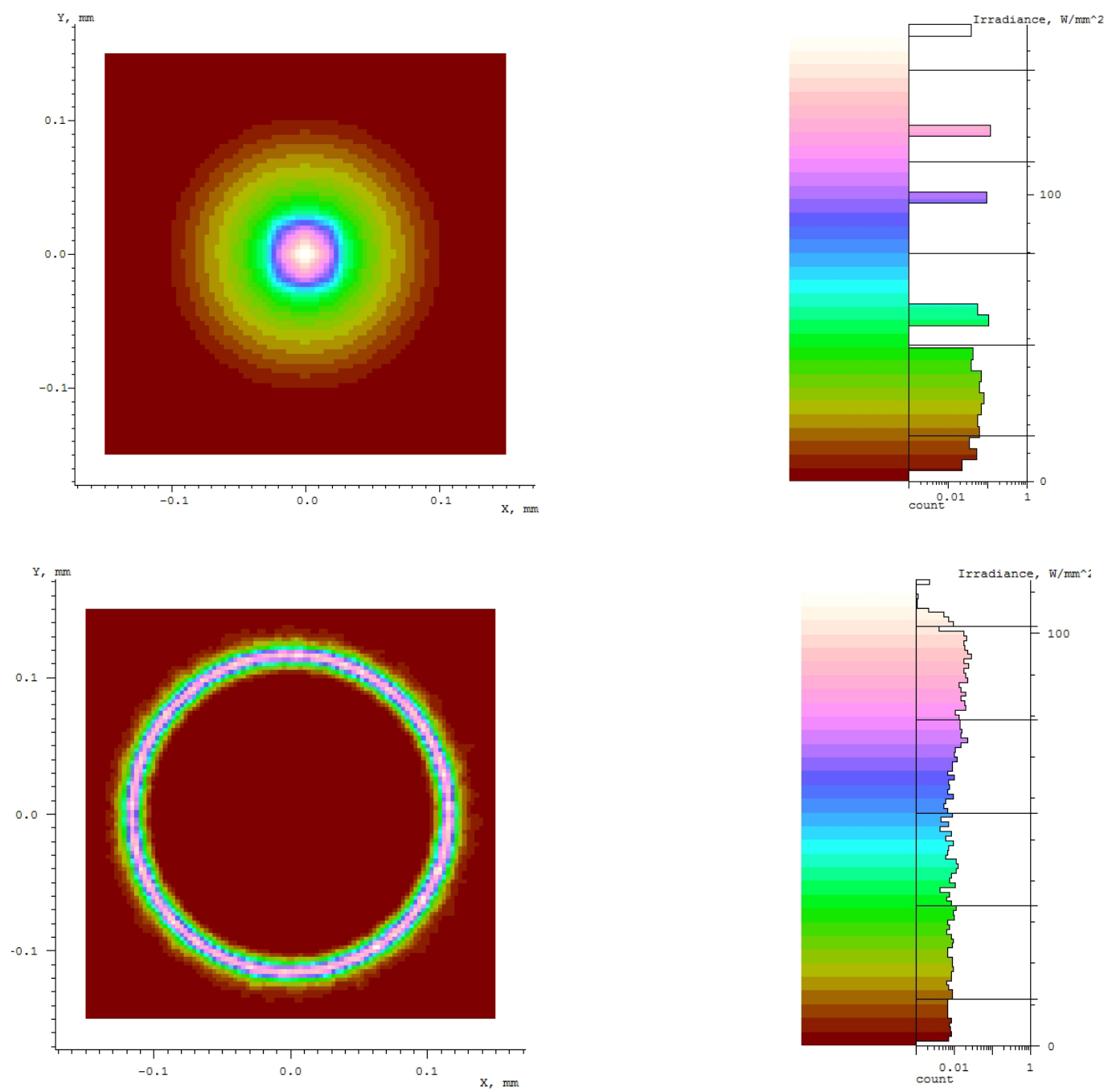

Figure 8 Illumination diagram at the receiving plane, for square dimensions $0.15 x 0.15 \mathrm{~mm}^{2}$. Top: revolution symmetry design. Bottom: spiral symmetry design, with its characteristic ring-shaped illumination pattern.

\section{SOME SPIRAL SYMMETRY COLLIMATOR DESIGNS}

The flat Fresnel lens is a simple and canonical good example to explain our designing philosophy. Nevertheless we will show here two more examples also developed with spiral symmetry, without a so detailed explanation of the design procedure. The first of these examples is a dome-shaped Fresnel lens collimator with minimum angular range output, while the second one is a similar collimator but presenting a $+/-30^{\circ}$ output. Both designs' simulations will be presented for PMMA material for the lens. 


\subsection{Dome-shaped Fresnel lens collimator with conical mirror}

This device was first proposed in [6] using however a thick continuous lens. This device, in an ideal situation (point source), can collimate completely a lambertian light source. Intensity patterns for both designs (continuous lens and Fresnel lens with spiral symmetry) are almost identical but, as stated in the introduction, optical absorption in the first case will be much higher than in the latter one (spiral design intensity shown in Figure 9). This is due to the obvious difference between both lens thicknesses. Optical efficiency of this spiral design is $81.1 \%$ for monochromatic $\lambda=555 \mathrm{~nm}$ simulation, and with a $100 \%$ reflectivity for the mirror.

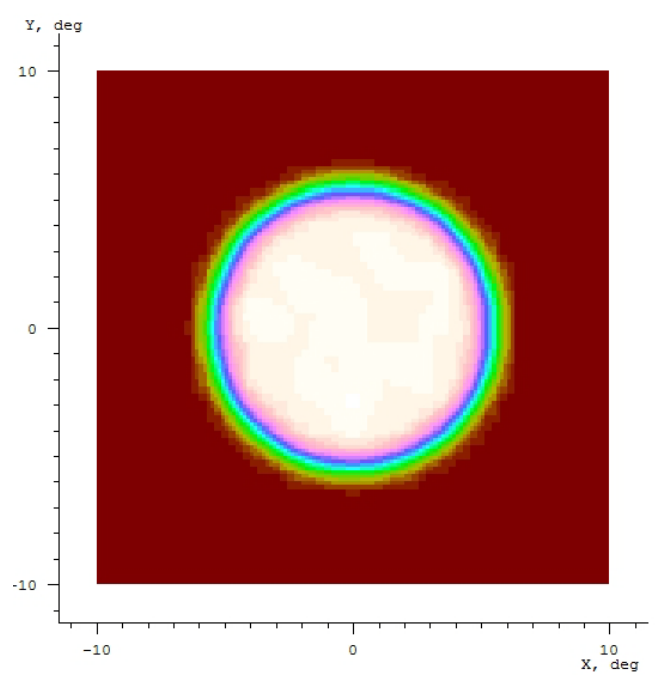

Figure 9 Intensity pattern for spiral symmetry collimator

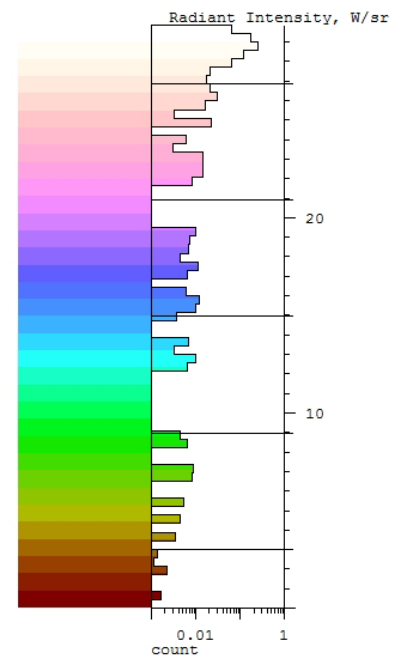

count

\subsection{Dome-shaped Fresnel lens collimator with conical mirror, $+/-30^{\circ}$ output angle}

This is a very similar design to that of previous section. Nevertheless there is a main difference in the output bundle angle: while the previous design tried to maximize bundle collimation, this one reduces to a $+/-30^{\circ}$ maximum angle the output bundle. Figure 10 shows intensity results in the far field for this spiral symmetry design. Results are similar to those that we would expect for a revolution symmetry design, with a neat $+/-30^{\circ}$ output bundle. Optical efficiency for this case is quite similar to the previous design (polychromatic simulation with mirror perfect reflectivity): $78.4 \%$.
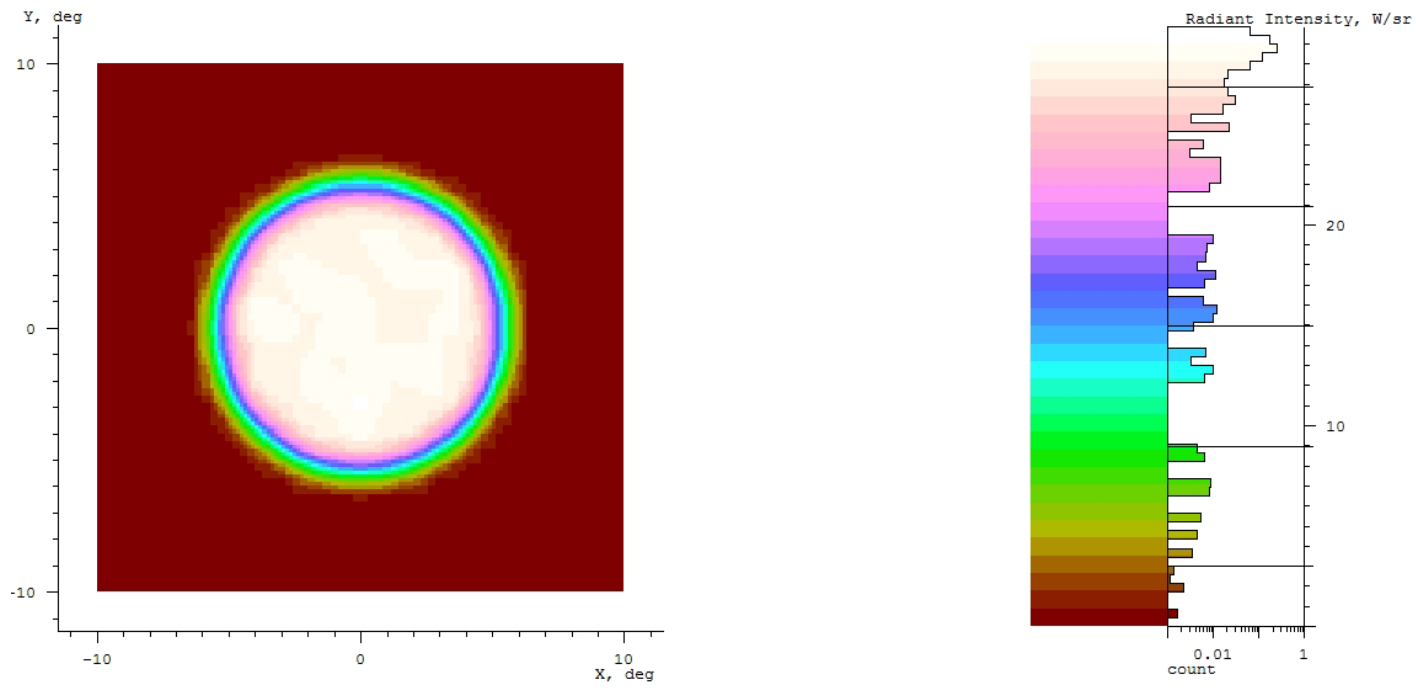

Figure $10+/-30^{\circ}$ collimator intensity diagram in the far field. 


\section{CONCLUSIONS}

A novel designing method for faceted elements, especially for Fresnel lenses, has been described in this manuscript. The designs explained along the text allow for an easy manufacturing process preventing from usual Fresnel lens fabrication problems. These problems, as explained in the introduction, are draft angles and manufacturing of pieces based on joining several parts.

The design method has been exposed in a neat and detailed way with a basic example as a flat Fresnel lens is. For this spiral symmetry performance differences between it and its corresponding "revolution symmetry" version have been described, explaining spiral version superiority. Other useful and interesting designs have been described, having based them in the design philosophy stated to explain the flat Fresnel lens.

\section{ACKNOWLEDGEMENTS}

Authors wish to thank the Spanish Ministries MCINN (ENGINEERING METAMATERIALS: CSD2008-00066, DEFFIO: TEC2008-03773, SIGMASOLES: PSS-440000-2009-30), MITYC (ECOLUX: TSI-020100-2010-1131, SEM: TSI-020302-2010-65), the Madrid Regional Government (SPIR: 50/2010O.23/12/09,TIC2010 and O-PRO: PIE/209/2010) and UPM (Q090935C59), and the EC, Spanish Ministry MITYC and C.A.M. under projects SSL4EU (Grant agreement $\mathrm{n}{ }^{\circ} 257550$, FP7/2007-2013), V-SS and SMS-IMAGING (TSI-020100-2011-445 and 372, Plan Avanza 2011) and 4LL (PIE/468/2010), respectively, for the support given in the preparation of the present work.

\section{REFERENCES}

[1] Leutz, R., Suzuki, A., [Nonimaging Fresnel lenses], Springer-Verlag, Berlin (2001).

[2] Miñano, J. C., "Application of the conservation of étendue theorem for 2-D subdomains of the phase space in nonimaging concentrators”, Appl. Optics, 23, 2021 (1984).

[3] Benítez, P., Mohedano, R., Miñano., J. C., "Design in 3D geometry with the Simultaneous Multiple Surface Design method of Nonimaging Optics”, Proc. SPIE, 3781, 12 (1999).

[4] Chaves, J., [Introduction to nonimaging optics], CRC Press, Boca Raton, 420-429 (2008).

[5] Winston, R., Miñano, J. C., Benítez, P., [Nonimaging Optics], Elsevier-Academic Press, New York (2005).

[6] Chaves, J., Falicoff, W., Sun, Y., Parkyn, B., "Simple optics that produce constant illuminance on a distant target”, Proc. SPIE 5529, 166 (2004). 